\title{
On the Adjacency, Laplacian, and Signless Laplacian Spectrum of Coalescence of Complete Graphs
}

\author{
S. R. Jog ${ }^{1}$ and Raju Kotambari ${ }^{2}$ \\ ${ }^{1}$ Gogte Institute of Technology, Udyambag, Belagavi, Karnataka 59008, India \\ ${ }^{2}$ Jain College of Engg, Machhe, Belagavi, Karnataka 590014, India \\ Correspondence should be addressed to S. R. Jog; sudhirrjog@yahoo.co.in
}

Received 23 June 2016; Accepted 3 August 2016

Academic Editor: Hong J. Lai

Copyright (C) 2016 S. R. Jog and R. Kotambari. This is an open access article distributed under the Creative Commons Attribution License, which permits unrestricted use, distribution, and reproduction in any medium, provided the original work is properly cited.

Coalescence as one of the operations on a pair of graphs is significant due to its simple form of chromatic polynomial. The adjacency matrix, Laplacian matrix, and signless Laplacian matrix are common matrices usually considered for discussion under spectral graph theory. In this paper, we compute adjacency, Laplacian, and signless Laplacian energy ( $Q$ energy) of coalescence of pair of complete graphs. Also, as an application, we obtain the adjacency energy of subdivision graph and line graph of coalescence from its $Q$ energy.

\section{Introduction}

Throughout the discussion by a graph we mean simple graph without self loops or multiple edges. Let $G$ be a simple graph on $n$ vertices with vertex set $\left[v_{1}, v_{2}, \ldots, v_{n}\right]$. The line graph of a graph $G$ is the graph with vertex set as edge set of $G$ with two vertices (edges of $G$ ) adjacent if and only if they are having a vertex in common. Similarly, the subdivision graph of a graph $G$ is the graph $S(G)$ obtained by inserting a vertex of degree two in each edge of $G$. The adjacency matrix of $G$ denoted by $A(G)$ is a matrix $A(G)=$ $\left[a_{i j}\right]$, where $a_{i j}=1$ if vertex $v_{i}$ is adjacent to $v_{j}$ and 0 otherwise. Clearly, $A(G)$ is real symmetric so that eigenvalues of $A(G)$ which are roots of its characteristic equation given by $P(G ; \lambda)=|\lambda I-A(G)|=0$ are real. They are denoted by $\lambda_{1}, \lambda_{2}, \ldots, \lambda_{n}$ and can be arranged in descending order as $\lambda_{1} \geq \lambda_{2} \geq \cdots \geq \lambda_{n}$. The spectrum of $G$ is collection of eigenvalues along with their multiplicity and energy of a graph is simply defined as $\sum\left|\lambda_{i}\right|$. For more details and rigorous treatment on adjacency spectra and energy, see [14]. Let $d_{i}$ denote the degree of a vertex $v_{i}$ which is the number of edges incident on it. The degree matrix $D$ is a diagonal matrix having diagonal entry as the degree of the corresponding vertex. We denote the average degree of a graph $G$, as avd $(G)=$ twice the no edges of $G /$ no. of vertices of $G$.
The matrix $C(G)=D(G)-A(G)$ is called Laplacian matrix. The roots of the characteristic polynomial of the Laplacian matrix are called Laplacian eigenvalues denoted by $\mu_{1}, \mu_{2}, \ldots, \mu_{n}$. The matrix $C(G)$ is also real symmetric but singular so eigenvalues can be arranged as $\mu_{1} \geq \mu_{2} \geq \cdots$ $\geq \mu_{n}=0$. The Laplacian spectrum of $G$ is the collection of Laplacian eigenvalues along with their multiplicity and Laplacian energy is defined as $E_{\mathrm{L}}(G)=\left|\sum \mu_{i}-\operatorname{avd}(G)\right|$.

For an extensive literature on Laplacian spectra and energy, one can refer to [5-11]. On similar lines, the signless Laplacian matrix of a graph $G$ is defined as $Q(G)=D(G)+$ $A(G)$. The signless Laplacian eigenvalues are also real and can be denoted by $v_{1}, v_{2}, \ldots, v_{n}$. The signless Laplacian energy (or simply $Q$ energy) is defined similar to Laplacian energy as $E_{\mathrm{SL}}(G)=\left|\sum v_{i}-\operatorname{avd}(G)\right|$.

Some results on signless Laplacian energy are available in [12-15]. The Laplacian and signless Laplacian eigenvalues for a connected graph are nonnegative.

Let $H_{1}$ and $H_{2}$ be graphs on disjoint sets of vertices, respectively. Suppose $U=\left\{u_{1}, u_{2}, \ldots, u_{t}\right\}$ is a clique in $H_{1}$ and $W=\left\{w_{1}, w_{2}, \ldots, w_{t}\right\}$ is a clique in $H_{2}$. Let $G$ be a graph obtained from $\mathrm{H}_{1}$ and $\mathrm{H}_{2}$ by identifying (coalescing into a single vertex) $u_{i}$ and $w_{i} 1 \leq i \leq t$. Then, $G$ is an overlap of $H_{1}$ and $H_{2}$ in $K_{t}$. It may be viewed as generalized coalescence denoted by $\mathrm{H}_{1} \circ \mathrm{H}_{2}$. 
The structure of $H_{1} \circ H_{2}$ depends on vertices chosen for overlap. Its chromatic polynomial can be split into chromatic polynomials of $H_{1}$ and $H_{2}$ (see [16]). If $t=1$, we call it vertex coalescence denoted by $H_{1}{ }^{\circ}{ }_{v} H_{2}$ and for $t=2$ we call it edge coalescence denoted by $\mathrm{H}_{1}{ }^{\circ} \mathrm{H}_{2}$. For vertex coalescence of two graphs $H_{1}$ and $H_{2}$, the adjacency matrix has the form

$$
A\left(H_{1} \circ{ }_{v} H_{2}\right)=\left[\begin{array}{ccc}
0 & J & J \\
J^{T} & A\left(H_{1}-u\right) & O \\
J^{T} & O & A\left(H_{2}-v\right)
\end{array}\right] .
$$

Similarly, the edge coalescence of two graphs $G_{1}$ and $G_{2}$ has the adjacency matrix structure

$$
A\left(H_{1}{ }^{\circ} e_{2} H_{2}\right)=\left[\begin{array}{ccc}
A\left(K_{2}\right) & J & J \\
J^{T} & A\left(H_{1}-e\right) & O \\
J^{T} & O & A\left(H_{2}-e\right)
\end{array}\right],
$$

where $J$ is matrix of all l's and $O$ is the matrix of all zeros having appropriate order.

\section{Results}

\subsection{Adjacency Energy}

Theorem 1. The energy (adjacency energy) of $K_{m}{ }^{\circ} K_{n}$ is given by $E\left[K_{m}{ }^{\circ}{ }_{v} K_{n}\right]=(m+n-4)+|\alpha|+|\beta|+|\delta|$, where $\alpha, \beta$, and $\delta$ are the roots of the cubic:

$$
\begin{gathered}
x^{3}-(m+n-4) x^{2}+(m n-3 m-3 n+6) x \\
+(2 m n-3 m-3 n+4)=0 .
\end{gathered}
$$

Proof. The coalescence of the complete graphs $K_{m}$ and $K_{n}$ at a point results in a graph with $m+n-1$ vertices and $\left(m C_{2}+n C_{2}\right)$ edges. The adjacency matrix takes the form

$$
A\left(K_{m{ }^{\circ}{ }_{v}} K_{n}\right)=\left[\begin{array}{ccc}
0 & J_{1 \times m-1} & J_{1 \times n-1} \\
J_{m-1 \times 1} & A\left(K_{m-1}\right) & O \\
J_{n-1 \times 1} & O & A\left(K_{n-1}\right)
\end{array}\right]
$$

so that characteristic polynomial is

$$
\begin{aligned}
P & {\left[K_{\left.m{ }^{\circ}{ }_{v} K_{n}: \lambda\right]=\mid \lambda I-A\left(K_{m}{ }^{\circ}{ }_{\nu} K_{n}\right)}\right.} \\
& =\left|\begin{array}{ccc}
\lambda & -J_{1 \times m-1} & -J_{1 \times n-1} \\
-J_{m-1 \times 1} & \lambda I_{m-1}-A\left(K_{m-1}\right) & O \\
-J_{n-1 \times 1} & O & \lambda I_{n-1}-A\left(K_{n-1}\right)
\end{array}\right| .
\end{aligned}
$$

By performing $C_{1}+\sum_{i=2}^{m-1} C_{i} /(\lambda-(m-2))$ and $C_{1}+$ $\sum_{j=m+1}^{m+n-1} C_{j} /(\lambda-(n-2))$ in succession, we have

$$
\begin{aligned}
P & {\left[K_{m{ }^{\circ}{ }^{\circ}} K_{n}: \lambda\right]=\left(\lambda-\frac{m-1}{\lambda-(m-2)}-\frac{n-1}{\lambda-(n-2)}\right) } \\
& \cdot P\left[K_{m-1}: \lambda\right] P\left[K_{n-1}: \lambda\right] \\
& =\left(\lambda-\frac{m-1}{\lambda-(m-2)}-\frac{n-1}{\lambda-(n-2)}\right)[\lambda-(m-2)] \\
& \cdot(\lambda+1)^{m-2}[\lambda-(n-2)](\lambda+1)^{n-2} .
\end{aligned}
$$

On simplifying, finally we get

$$
\begin{aligned}
P & {\left[K_{m{ }^{\circ}{ }_{\nu}} K_{n}: \lambda\right]:(\lambda+1)^{m+n-4}\left[\lambda^{3}-(m+n-4) \lambda^{2}\right.} \\
& +(m n-3 m-3 n+6) \lambda+(m-1)(n-2) \\
& +(n-1)(m-2)] .
\end{aligned}
$$

From this equation, the theorem follows.

Theorem 2. The energy (adjacency energy) of edge coalescence $\left(K_{m}{ }^{\circ} K_{n}\right)$ of complete graphs is given by $E\left[K_{m}{ }^{\circ} K_{n}\right]=(m+n-$ $5)+|\alpha|+|\beta|+|\delta|$, where $\alpha, \beta$, and $\delta$ are the roots of the cubic equation:

$$
\begin{gathered}
x^{3}-(m+n-5) x^{2}+(m n-4 m-4 n+11) x \\
+(3 m n-7 m-7 n+15)=0 .
\end{gathered}
$$

Proof. The coalescence of the complete graphs $K_{m}$ and $K_{n}$ on an edge results in a graph with $m+n-2$ vertices and $m C_{2}+$ $n C_{2}-1$ edges. The adjacency matrix takes the form

$$
A\left(K_{m}{ }^{\circ} K_{n}\right)=\left[\begin{array}{ccc}
A\left(K_{2}\right) & J_{2 \times m-2} & J_{2 \times n-2} \\
J_{m-2 \times 2} & A\left(K_{m}-e\right) & O \\
J_{n-2 \times 2} & O & A\left(K_{n}-e\right)
\end{array}\right]
$$

so that the characteristic polynomial of the edge coalescence is

$$
\begin{aligned}
P & {\left[K_{m}{ }^{\circ} K_{n}: \lambda\right]=\left|\lambda I-A\left(K_{m}{ }^{\circ} K_{n}\right)\right| } \\
& =\left|\begin{array}{ccc}
\lambda I-A\left(K_{2}\right) & -J_{2 \times m-2} & -J_{2 \times n-2} \\
-J_{m-2 \times 2} & \lambda I-A\left(K_{m}-e\right) & O \\
-J_{n-2 \times 2} & O & \lambda I-A\left(K_{n}-e\right)
\end{array}\right| .
\end{aligned}
$$

By performing $C_{j}+\sum_{i=3}^{m} C_{i} /(\lambda-m+3)$ for $j=1,2$ we have

$$
P\left[\left(K_{m}{ }^{\circ} K_{n}\right): \lambda\right]=\left|\begin{array}{cccc}
\lambda-\frac{m-2}{\lambda-m+3} & -1-\frac{m-2}{-m+3} & -J_{1 \times m-2} & -J_{1 \times n-2} \\
-1-\frac{m-2}{-m+3} & \lambda-\frac{m-2}{\lambda-m+3} & -J_{1 \times m-2} & -J_{1 \times n-2} \\
O & O & \lambda I-A\left(K_{m-2}\right) & O \\
-J_{n-2 \times 1} & -J_{n-2 \times 1} & O & \lambda I-A\left(K_{n-2}\right)
\end{array}\right|
$$


By performing $C_{j}+\sum_{i=m+1}^{m+n-2} C_{i} /(\lambda-n+3)$ for $j=1,2$ we have

$$
\begin{aligned}
P\left[\left(K_{m} \circ e_{n} K_{n}\right): \lambda\right] & =\left|\begin{array}{cccc}
\lambda-\frac{m-2}{\lambda-m+3}-\frac{n-2}{\lambda-n+3} & -1-\frac{m-2}{\lambda-m+3}-\frac{n-2}{\lambda-n+3} & -J_{1 \times m-2} & -J_{1 \times n-2} \\
-1-\frac{m-2}{\lambda-m+3}-\frac{n-2}{\lambda-n+3} & \lambda-\frac{m-2}{\lambda-m+3}-\frac{n-2}{\lambda-n+3} & -J_{1 \times n-2} & -J_{1 \times n-2} \\
O & O & \lambda I-A\left(K_{m-2}\right) & O \\
O & O & \lambda I-A\left(K_{n-2}\right)
\end{array}\right| \\
& =\left[\begin{array}{ccc}
\left.\lambda-\frac{m-2}{\lambda-m+3}-\frac{n-2}{\lambda-n+3}\right]^{2}-\left[-1-\frac{m-2}{\lambda-m+3}-\frac{n-2}{\lambda-n+3}\right]^{2} P\left[K_{m-2}: \lambda\right] P\left[K_{n-2}: \lambda\right] .
\end{array}\right.
\end{aligned}
$$

On expanding and simplifying, we get the required polynomial and hence the theorem.

2.2. Laplacian Energy. Now we discuss the Laplacian energy of coalescence.

Lemma 3 (see [17]). If $G$ is any connected graph of order $n$ with Laplacian eigenvalues $\mu_{1}, \mu_{2}, \ldots, \mu_{n}$ with $\mu_{n}=0$, then, the number of spanning trees of $G$ is given by

$$
\tau(G)=\frac{\mu_{1}, \mu_{2}, \ldots, \mu_{n-1}}{n} .
$$

Proof. The degree matrix of the vertex coalescence $K_{m}{ }^{\circ} K_{n}$ with suitable labeling has the form

Theorem 4. The Laplacian energy of the vertex coalescence of complete graphs $K_{m}$ and $K_{n}$ is given by

$$
E_{L}\left(K_{m} \circ K_{n}\right)=\frac{\left(3 m^{2}+3 n^{2}-5 m-5 n+1\right)}{(m+n-1)} .
$$

$$
D\left[K_{m} \circ K_{n}\right]=\left[\begin{array}{ccccccccc}
m+n-2 & 0 & \cdots & \cdots & 0 & 0 & 0 & \cdots & 0 \\
0 & m-1 & 0 & \cdots & 0 & 0 & 0 & \cdots & 0 \\
0 & 0 & m-1 & \cdots & 0 & 0 & 0 & \cdots & 0 \\
\vdots & \vdots & \vdots & \ddots & \vdots & \vdots & \vdots & \vdots & \vdots \\
0 & 0 & 0 & \cdots & m-1 & 0 & 0 & 0 & 0 \\
0 & 0 & 0 & \cdots & 0 & n-1 & 0 & 0 & 0 \\
0 & 0 & 0 & \cdots & 0 & 0 & n-1 & 0 & 0 \\
\vdots & \vdots & \vdots & \vdots & \vdots & \vdots & \vdots & \ddots & \vdots \\
0 & 0 & 0 & 0 & 0 & 0 & 0 & \cdots & n-1
\end{array}\right]
$$

The adjacency matrix is

$$
A\left(K_{m}{ }^{\circ} K_{n}\right)=\left[\begin{array}{ccc}
O & J & J \\
J^{T} & A\left[K_{m-1}\right] & O \\
J^{T} & O & A\left[K_{n-1}\right]
\end{array}\right] .
$$


The Laplacian matrix now becomes

$$
C\left(K_{m} \circ K_{n}\right)=\left[\begin{array}{ccccccccc}
m+n-2 & -1 & -1 & \cdots & -1 & -1 & -1 & \cdots & -1 \\
-1 & m-1 & -1 & \cdots & -1 & 0 & 0 & \cdots & 0 \\
-1 & -1 & m-1 & \cdots & -1 & 0 & 0 & \cdots & 0 \\
\vdots & \vdots & \vdots & \ddots & \vdots & \vdots & \vdots & \vdots & \vdots \\
-1 & -1 & -1 & \cdots & m-1 & 0 & 0 & \cdots & 0 \\
-1 & 0 & 0 & \cdots & 0 & n-1 & -1 & \cdots & -1 \\
-1 & 0 & 0 & \cdots & 0 & -1 & n-1 & \cdots & -1 \\
\vdots & \vdots & \vdots & \vdots & \vdots & \vdots & \vdots & \ddots & \vdots \\
-1 & 0 & 0 & 0 & 0 & -1 & -1 & \cdots & n-1
\end{array}\right]
$$

so that the Laplacian polynomial is

$$
\begin{aligned}
& \left|\mu I-C\left(K_{m}{ }^{\circ} K_{n}\right)\right| \\
& =\left|\begin{array}{ccccccccc}
\mu-(m+n-2) & 1 & 1 & \cdots & 1 & 1 & 1 & \cdots & 1 \\
1 & \mu-(m-1) & 1 & \cdots & 1 & 0 & 0 & \ldots & 0 \\
1 & 1 & \mu-(m-1) & \cdots & 1 & 0 & 0 & \ldots & 0 \\
\vdots & \vdots & \vdots & \ddots & \vdots & \vdots & \vdots & \vdots & \vdots \\
1 & 1 & 1 & \cdots & \mu-(m-1) & 0 & 0 & \ldots & 0 \\
1 & 0 & 0 & \cdots & 0 & \mu-(m-1) & 0 & 0 & 0 \\
1 & 0 & 0 & \cdots & 0 & 0 & \mu-(m-1) & 0 & 0 \\
1 & \vdots & \vdots & \vdots & \vdots & \vdots & \vdots & \ddots & \vdots
\end{array}\right| .
\end{aligned}
$$

Performing $C_{1}-(1 /(\mu-1)) \sum_{i=2}^{n} C_{i}$ we get

$$
\begin{aligned}
& \left|\mu I-C\left(K_{m}{ }^{\circ} K_{n}\right)\right| \\
& =\begin{array}{ccccccccc}
\mu-(m+n-2)-\frac{m-1}{\lambda-1} & 1 & 1 & \cdots & 1 & 1 & 1 & \cdots & 1 \\
1 & \mu-(m-1) & 1 & \cdots & 1 & 0 & 0 & \cdots & 0 \\
1 & 1 & \mu-(m-1) & \cdots & 1 & 0 & 0 & \cdots & 0 \\
\vdots & \vdots & \vdots & \ddots & \vdots & \vdots & \vdots & \vdots & \vdots \\
1 & 1 & 1 & \cdots & \mu-(m-1) & 0 & 0 & \cdots & 0 \\
1 & 0 & 0 & \ldots & 0 & \mu-(m-1) & 0 & 0 & 0 \\
1 & 0 & 0 & \ldots & 0 & 0 & \mu-(m-1) & 0 & 0 \\
1 & \vdots & \vdots & \vdots & \vdots & \vdots & \vdots & \ddots & \vdots \\
& 0 & 0 & 0 & 0 & 0 & 0 & \cdots & \mu-(m-1)
\end{array} \mid .
\end{aligned}
$$


Again performing $C_{1}-(1 /(\mu-1)) \sum_{i=m+1}^{m+n-1} C_{i}$ and directly expanding along first column, we get

$$
\begin{aligned}
& \left|\mu I-C\left(K_{m}{ }_{v} K_{n}\right)\right| \\
& \quad=\left[\mu-(m+n-2)-\frac{n-1}{\mu-1}-\frac{m-1}{\mu-1}\right] \\
& \cdot L\left[K_{m-1}: \lambda\right] L\left[K_{n-1}: \lambda\right]=\mu[\mu-(m+n-1)] \\
& \cdot(\mu-1)(\mu-m)^{m-2}(\mu-n)^{n-2} .
\end{aligned}
$$

So that the Laplacian spectrum is $S_{\mathrm{L}}\left(K_{m}{ }^{\circ} K_{n}\right)=m, m-$ 2 times, $n, n-2$ times, $m+n-1,1$, and 0 .

Now the $\operatorname{avd}\left(K_{m}{ }^{\circ} K_{n}\right)=(m(m-1)+n(n-1)) /(m+n-1) ;$ hence, the Laplacian energy becomes

$$
\begin{aligned}
E_{\mathrm{L}}\left(K_{m}{ }_{v} K_{n}\right)=\left|\sum_{i=1}^{m+n-1} \mu_{i}-\frac{m(m-1)+n(n-1)}{m+n-1}\right| \\
=\left|m-\frac{m(m-1)+n(n-1)}{m+n-1}\right|(m-2) \\
+\left|n-\frac{m(m-1)+n(n-1)}{m+n-1}\right|(n-2) \\
+\left|m+n-1-\frac{m(m-1)+n(n-1)}{m+n-1}\right| \\
+\left|1-\frac{m(m-1)+n(n-1)}{m+n-1}\right| \\
+\left|0-\frac{m(m-1)+n(n-1)}{m+n-1}\right| \\
=\frac{3 m^{2}+3 n^{2}-5 m-5 n+1}{m+n-1} .
\end{aligned}
$$

Corollary 5. The number of spanning trees of $K_{m}{ }^{\circ} K_{n}$ according to Lemma 3 is $\tau\left(K_{m}{ }^{\circ} K_{n}\right)=m(m-2) n(n-2)(m+n-$ $1) /(m+n-1)=m(m-2) n(n-2)$ as expected since $K_{m}{ }_{v} K_{n}$ has a cut point with number of spanning trees $m^{m-2}$ and $n^{n-2}$ in each block (complete graph) separately.

Theorem 6. The Laplacian energy of the edge coalescence of complete graphs $K_{m}$ and $K_{n}$ is given by

$$
\begin{aligned}
E_{L}\left(K_{m} \circ{ }_{e} K_{n}\right)= & \left|\frac{(m-n)(m-1)+2}{m+n-2}\right|(m-3) \\
& +\left|\frac{(n-m)(m-1)+2}{m+n-2}\right|(n-3) \\
& +\left|\alpha-\frac{m^{2}+n^{2}-m-n-2}{m+n-2}\right| \\
& +\left|\beta-\frac{m^{2}+n^{2}-m-n-2}{m+n-2}\right| \\
& +\left|\delta-\frac{m^{2}+n^{2}-m-n-2}{m+n-2}\right|,
\end{aligned}
$$

where $\alpha, \beta$, and $\delta$ are the roots of the cubic equation:

$$
\begin{gathered}
x^{3}-2(m+n-1) x^{2}+\left(m^{2}+n^{2}+2 m n-4\right) x \\
-2(m+n-2)^{2}=0 .
\end{gathered}
$$

Proof. The degree matrix of the edge coalescence $\left(K_{m}{ }^{\circ} K_{n}\right)$ with suitable labeling has the form

$$
D\left(K_{m}{ }_{0} K_{n}\right)=\left[\begin{array}{ccccccccc}
m+n-3 & 0 & \cdots & \cdots & 0 & 0 & 0 & \cdots & 0 \\
0 & m+n-3 & 0 & \cdots & 0 & 0 & 0 & \cdots & 0 \\
0 & 0 & m-1 & \cdots & 0 & 0 & 0 & \cdots & 0 \\
\vdots & \vdots & \vdots & \ddots & \vdots & \vdots & \vdots & \vdots & \vdots \\
0 & 0 & 0 & \cdots & m-1 & 0 & 0 & 0 & 0 \\
0 & 0 & 0 & \cdots & 0 & n-1 & 0 & 0 & 0 \\
0 & 0 & 0 & \cdots & 0 & 0 & n-1 & 0 & 0 \\
\vdots & \vdots & \vdots & \vdots & \vdots & \vdots & \vdots & \ddots & \vdots \\
0 & 0 & 0 & 0 & 0 & 0 & 0 & \cdots & n-1
\end{array}\right]
$$

The adjacency matrix is

$$
A\left(K_{m}{ }^{\circ} K_{n}\right)=\left[\begin{array}{cccc}
O & 1 & J_{1 \times m-2} & J_{1 \times n-2} \\
1 & 0 & J_{1 \times m-2} & J_{1 \times n-2} \\
J_{m-2 \times 1} & J_{m-2 \times 1} & A\left(K_{m-2}\right) & O \\
J_{n-2 \times 1} & J_{n-2 \times 1} & O & A\left(K_{n-2}\right)
\end{array}\right] .
$$




$$
C\left(K_{m} \circ K_{n}\right)=\left[\begin{array}{ccccccccc}
m+n-3 & -1 & -1 & \cdots & -1 & -1 & -1 & \cdots & -1 \\
-1 & m+n-3 & -1 & \cdots & -1 & -1 & -1 & \cdots & -1 \\
-1 & -1 & m-1 & \cdots & -1 & 0 & 0 & \cdots & 0 \\
\vdots & \vdots & \vdots & \ddots & \vdots & \vdots & \vdots & \vdots & \vdots \\
-1 & -1 & -1 & \cdots & m-1 & 0 & 0 & \cdots & 0 \\
-1 & -1 & 0 & \cdots & 0 & n-1 & -1 & \cdots & -1 \\
-1 & -1 & 0 & \cdots & 0 & -1 & n-1 & \cdots & -1 \\
\vdots & \vdots & \vdots & \vdots & \vdots & \vdots & \vdots & \ddots & \vdots \\
-1 & -1 & 0 & \cdots & 0 & -1 & -1 & \cdots & n-1
\end{array}\right]
$$

The Laplacian polynomial is then given by

$$
\begin{aligned}
& \left|\mu I-C\left(K_{m}^{\circ} e K_{n}\right)\right|
\end{aligned}
$$

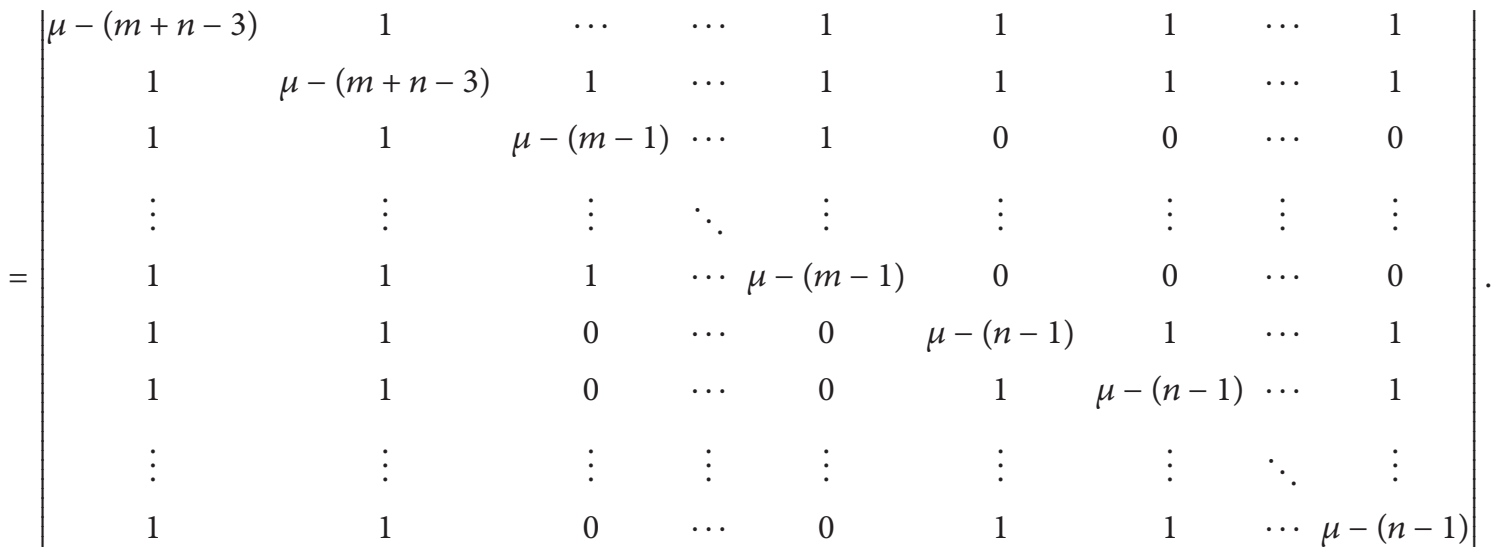

Performing $C_{1}-(1 /(\mu-2)) \sum_{i=3}^{m} C_{i}$ and $C_{2}-(1 /(\mu-$ 2)) $\sum_{i=3}^{m} C_{i}$,

$$
\begin{aligned}
& \left|\mu I-C\left(K_{m}{ }^{\circ} K_{n}\right)\right|
\end{aligned}
$$

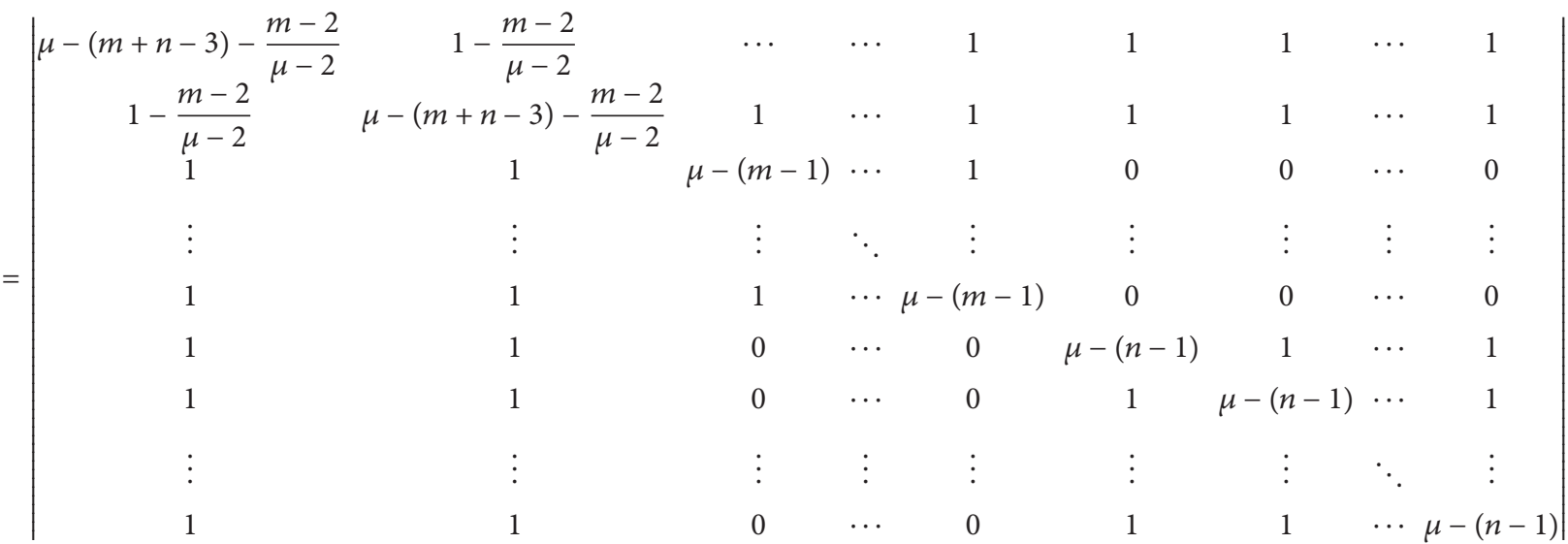


Again performing $C_{1}-(1 /(\mu-2)) \sum_{i=m+1}^{m+n-2} C_{i}$ and $C_{2}-$ $(1 /(\mu-2)) \sum_{i=m+1}^{m+n-2} C_{i}$ and directly expanding give

$$
\left|\mu I-C\left(K_{m} \circ e_{n}\right)\right|=\left|\begin{array}{cc}
\mu-(m+n-3)-\frac{m+n-4}{\mu-2} & 1-\frac{m+n-4}{\mu-2} \\
1-\frac{m+n-4}{\mu-2} & \mu-(m+n-3)-\frac{m+n-4}{\mu-2}
\end{array}\right| L\left[K_{m-2}: \mu\right] L\left[K_{n-2}: \mu\right] .
$$

On simplifying, we get the Laplacian polynomial as

$$
\begin{aligned}
& \left|\mu I-C\left(K_{m} \circ e K_{n}\right)\right|=\mu(\mu-m)^{m-3}(\mu-n)^{n-3}\left[\mu^{3}\right. \\
& -2(m+n-1) \mu^{2}+\left(m^{2}+n^{2}+2 m n-4\right) \mu \\
& \left.-2(m+n-2)^{2}\right]
\end{aligned}
$$

On equating to zero and extracting eigenvalues from the equation above, the theorem follows.

Note. When $m=n$, the Laplacian polynomial is

$$
\begin{aligned}
& \left|\mu I-C\left(K_{n} \circ e_{n}\right)\right|=(\mu-n)^{2 n-3} \\
& \quad \cdot\left[\mu^{3}-2(2 n-1) \mu^{2}+\left(4 n^{2}-4\right) \mu-8(n-1)^{2}\right] \\
& \quad=(\mu-n)^{2 n-3}[\mu-2]\left[\mu-2(n-1)^{2}\right] .
\end{aligned}
$$

The Laplacian eigenvalues are $n, 2 n-3$ times, $2(n-1)$ twice, 2 , and 0 once.

Since $\operatorname{adg}\left(K_{m} \circ K_{n}\right)=\left(n^{2}-n-1\right) /(2 n-2)$ the Laplacian energy is

$$
\begin{aligned}
E_{\mathrm{L}}\left(K_{n} \circ_{e} K_{n}\right)= & \left|0-\frac{n^{2}-n-1}{2 n-2}\right|+\left|2-\frac{n^{2}-n-1}{2 n-2}\right| \\
& +\left|n-\frac{n^{2}-n-1}{2 n-2}\right|(2 n-3)
\end{aligned}
$$

$E_{S L}\left(K_{m}{ }_{v} K_{n}\right)$

$=\frac{|m n-3 m-n+5|(m-2)+|m n-3 n-m+5|(n-2)+|2 m n-3.5 m-3.5 n+6+\sqrt{a}|+|2 m n-3.5 m+3.5 n+6-\sqrt{a}|+|2 m n-m-n+7|}{m+n-2}$,
2.3. Signless Laplacian Energy. Now we consider the case of signless Laplacian matrix of the coalescence of complete graphs and deduce the corresponding energy. Before we do so, consider the following results.

Lemma 7 (see [17]). If $G$ is any graph with $p$ vertices and $q$ edges, then characteristic polynomial of line graph $L(G)$ in terms of Q (signless Laplacian) polynomial is given by

$$
P[L(G): \lambda]=(\lambda+2)^{q-P} Q[G: \lambda+2] .
$$

Lemma 8 (see [18]). If $G$ is any graph with $p$ vertices and $q$ edges, then characteristic polynomial of subdivision graph $S(G)$ in terms of $Q$ (signless Laplacian) polynomial is given by

$$
P[S(G): \lambda]=\lambda^{q-p} Q\left[G: \lambda^{2}\right] .
$$

Theorem 9. The signless Laplacian energy of the vertex coalescence of complete graphs $K_{m}$ and $K_{n}$ is given by

where $a=(m-n)^{2}+m+n-1.75$. 
Proof. From the degree matrix and adjacency matrix of the vertex coalescence $\left(K_{m}{ }^{\circ} K_{n}\right)$, we have the signless Laplacian matrix:

$$
Q\left(K_{m} \circ K_{n}\right)=\left[\begin{array}{ccccccccc}
m+n-2 & 1 & \cdots & \cdots & 1 & 1 & 1 & \cdots & 1 \\
1 & m-1 & 1 & \cdots & 1 & 1 & 1 & \cdots & 1 \\
1 & 1 & m-1 & \cdots & 1 & 0 & 0 & \cdots & 0 \\
\vdots & \vdots & \vdots & \ddots & \vdots & \vdots & \vdots & \vdots & \vdots \\
1 & 1 & 1 & \cdots & m-1 & 0 & 0 & \cdots & 0 \\
1 & 0 & 0 & \cdots & 0 & n-1 & 1 & \cdots & 1 \\
1 & 0 & 0 & \cdots & 0 & 1 & n-1 & \cdots & 1 \\
\vdots & \vdots & \vdots & \vdots & \vdots & \vdots & \vdots & \ddots & \vdots \\
1 & 0 & 0 & \cdots & 0 & 1 & 1 & \cdots & n-1
\end{array}\right]
$$

The signless Laplacian polynomial is then

$$
\begin{aligned}
& \left|\nu I-Q\left(K_{m} \circ{ }_{v} K_{n}\right)\right| \\
& =\left(\begin{array}{ccccccccc}
\nu-(m+n-2) & -1 & -1 & \cdots & -1 & -1 & -1 & \ldots & -1 \\
-1 & v-(m-1) & -1 & \ldots & -1 & -1 & -1 & \ldots & -1 \\
-1 & -1 & v-(m-1) & \ldots & -1 & 0 & 0 & \ldots & 0 \\
\vdots & \vdots & \vdots & \ddots & \vdots & \vdots & \vdots & \vdots & \vdots \\
-1 & -1 & -1 & \cdots & v-(m-1) & 0 & 0 & \ldots & -1 \\
-1 & 0 & 0 & \cdots & 0 & v-(n-1) & -1 & \ldots & -1 \\
-1 & 0 & 0 & \cdots & 0 & -1 & v-(n-1) & \cdots & 0 \\
\vdots & \vdots & \vdots & \vdots & \vdots & \vdots & \vdots & \ddots & \vdots \\
-1 & 0 & 0 & \ldots & 0 & -1 & -1 & \cdots & v-(n-1)
\end{array} \mid .\right.
\end{aligned}
$$

Performing $C_{1}+(1 /(\nu-2 m+3)) \sum_{i=2}^{n} C_{i}$,

$$
\begin{aligned}
& \left|\nu I-Q\left(K_{m}{ }_{\nu} K_{n}\right)\right|
\end{aligned}
$$

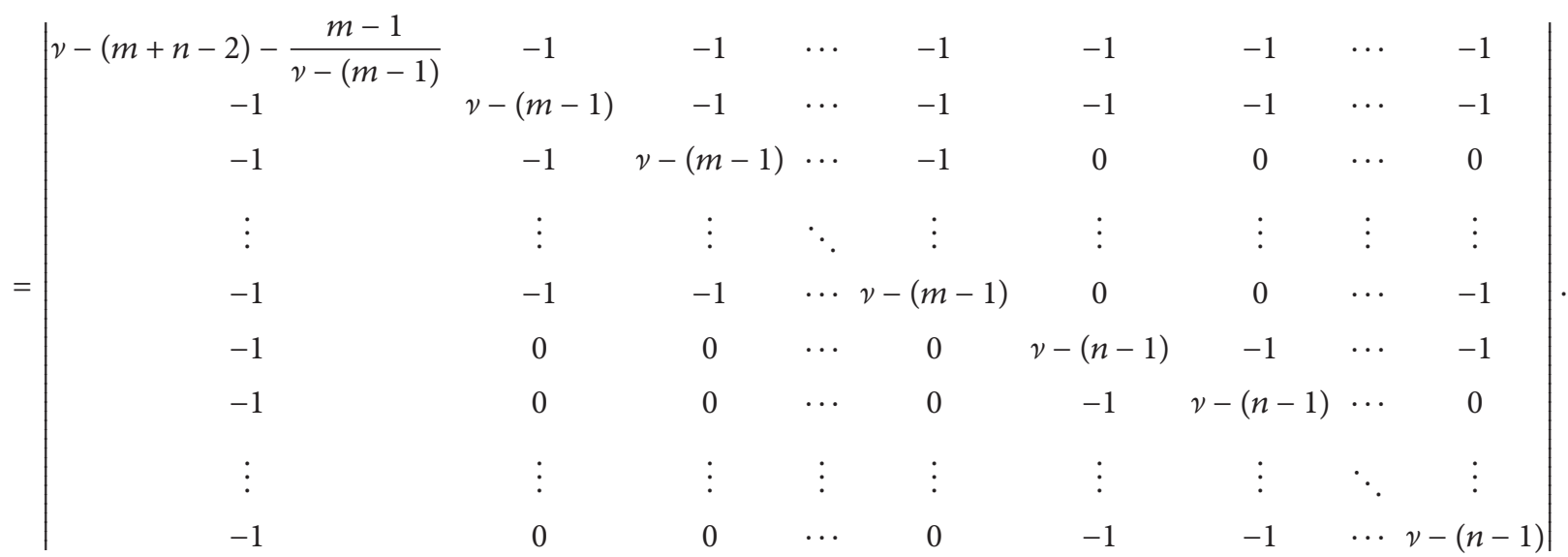


Again performing $C_{1}+(1 /(\nu-2 m+3)) \sum_{j=m+1}^{m+n-1} C_{j}$ then directly expanding along first column, we obtain

$$
\begin{aligned}
\left|v I-Q\left(K_{m} \circ{ }_{v} K_{n}\right)\right|=[v-(m+n-3)] \\
\cdot[v-(m-2)]^{m-2}[v-(n-2)]^{n-2} \\
\cdot\left[v^{2}-(2 m+2 n-5) v+4 m n-6 m-6 n+8\right] .
\end{aligned}
$$

The signless Laplacian eigenvalues are $(m-2)(m-2)$ times, $(n-2)(n-2)$ times, $(m+n-2.5) \pm$ $\sqrt{(m-n)^{2}+m+n-1.75}$, and $m+n-3$.

Now the $\operatorname{adg}\left(K_{m} \circ e K_{n}\right)=(m(m-1)+n(n-1)-1) /(m+$ $n-2)$; hence, the theorem follows.

Corollary 10. From Lemma 7, the energy (adjacency energy) of line graph of $K_{m}{ }^{\circ} K_{n}$ is given by

$$
\begin{aligned}
E\left[L\left(K_{m} \circ{ }_{v} K_{n}\right)\right]= & \left(m^{2}+n^{2}-3 m-3 n+2\right) \\
& +(m-2)|m-4|+(n-2)|n-4| \\
& +(2 m+2 n-9) \quad m, n \geq 3 .
\end{aligned}
$$

In particular for $m, n \geq 4$,

$$
\begin{aligned}
E\left[L\left(K_{m} \circ{ }_{v} K_{n}\right)\right]= & 2(m-2)^{2}+2(n-2)^{2}-3 \\
& +2 \sqrt{(m-n)^{2}+m+n-1.75}
\end{aligned}
$$

Corollary 11. From Lemma 8, the energy (adjacency) of subdivision graph of $K_{m}{ }^{\circ} K_{n}$ where $m, n \geq 2$, is given by

$$
\begin{aligned}
E\left[S\left(K_{m} \circ{ }_{v} K_{n}\right)\right]= & 2 \sqrt{m+n-3}+2 \sqrt{m-2}(m-2) \\
& +2 \sqrt{n-2}(n-2)
\end{aligned}
$$

$$
\begin{aligned}
& +\sqrt{2} \sqrt{(2 m+2 n-5)} \\
& \pm 2 \sqrt{m^{2}+n^{2}-11 m-11 n-8} .
\end{aligned}
$$

Theorem 12. The signless Laplacian energy of the edge coalescence of complete graphs $K_{m}$ and $K_{n}$ is given by

$$
\begin{aligned}
E_{S L}\left(K_{m}{ }_{0} K_{n}\right)= & \left|\frac{2 m n-5 m-5 n+10}{m+n-2}\right|(m+n-2) \\
& +\left|\frac{m n-n^{2}-3 m-n+6}{m+n-2}\right|(m-3) \\
& +\left|\frac{m n-m^{2}-3 n-m+6}{m+n-2}\right|(n-3) \\
& +\left|\alpha-\frac{m^{2}+n^{2}-m-n-2}{m+n-2}\right| \\
& +\left|\beta-\frac{m^{2}+n^{2}-m-n-2}{m+n-2}\right| \\
& +\left|\delta-\frac{m^{2}+n^{2}-m-n-2}{m+n-2}\right|,
\end{aligned}
$$

where $\alpha, \beta$, and $\delta$ are roots of the cubic equation:

$$
\begin{aligned}
x^{3}- & (3 m+3 n-10) x^{2} \\
& +2\left(m^{2}+n^{2}+4 m n-11 m-11 n+20\right) x \\
& -4(m+n-4)(m-2)(n-2)=0 .
\end{aligned}
$$

Proof. From the degree and adjacency matrix, the signless Laplacian matrix of the edge coalescence is

$$
Q\left(K_{m}{ }^{\circ} K_{n}\right)=\left[\begin{array}{ccccccccc}
m+n-3 & 1 & \cdots & \cdots & 1 & 1 & 1 & \cdots & 1 \\
1 & m+n-3 & 1 & \cdots & 1 & 1 & 1 & \cdots & 1 \\
1 & 1 & m-1 & \cdots & 1 & 0 & 0 & \cdots & 0 \\
\vdots & \vdots & \vdots & \ddots & \vdots & \vdots & \vdots & \vdots & \vdots \\
1 & 1 & 1 & \cdots & m-1 & 0 & 0 & \cdots & 0 \\
1 & 1 & 0 & \cdots & 0 & n-1 & 1 & \cdots & 1 \\
1 & 1 & 0 & \cdots & 0 & 1 & n-1 & \cdots & 1 \\
\vdots & \vdots & \vdots & \vdots & \vdots & \vdots & \vdots & \ddots & \vdots \\
1 & 1 & 0 & \cdots & 0 & 1 & 1 & \cdots & n-1
\end{array}\right] .
$$


The signless Laplacian polynomial is then given by

$$
\begin{aligned}
& \left|\nu I-Q\left(K_{m} \circ e_{n}\right)\right|
\end{aligned}
$$

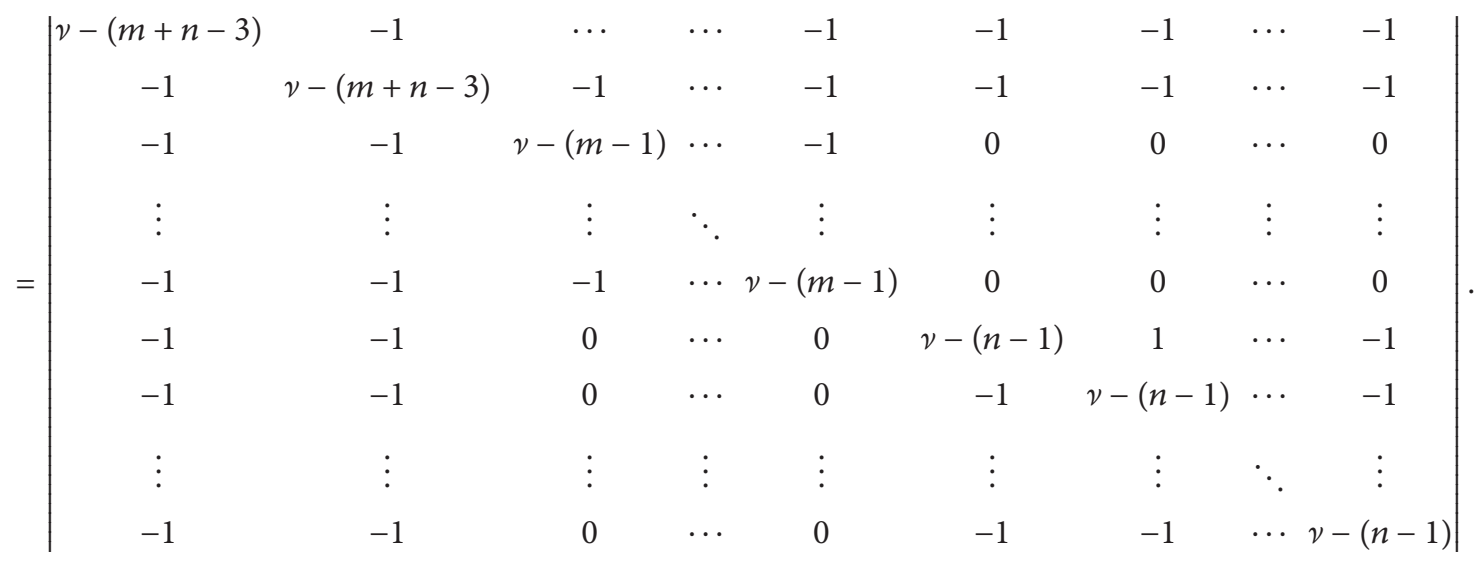

Performing $C_{1}, C_{2}+(1 /(\nu-2 m+4)) \sum_{i=3}^{m} C_{i}$,

$$
\begin{aligned}
& \left|\nu I-Q\left(K_{m}{ }^{\circ} K_{n}\right)\right|
\end{aligned}
$$

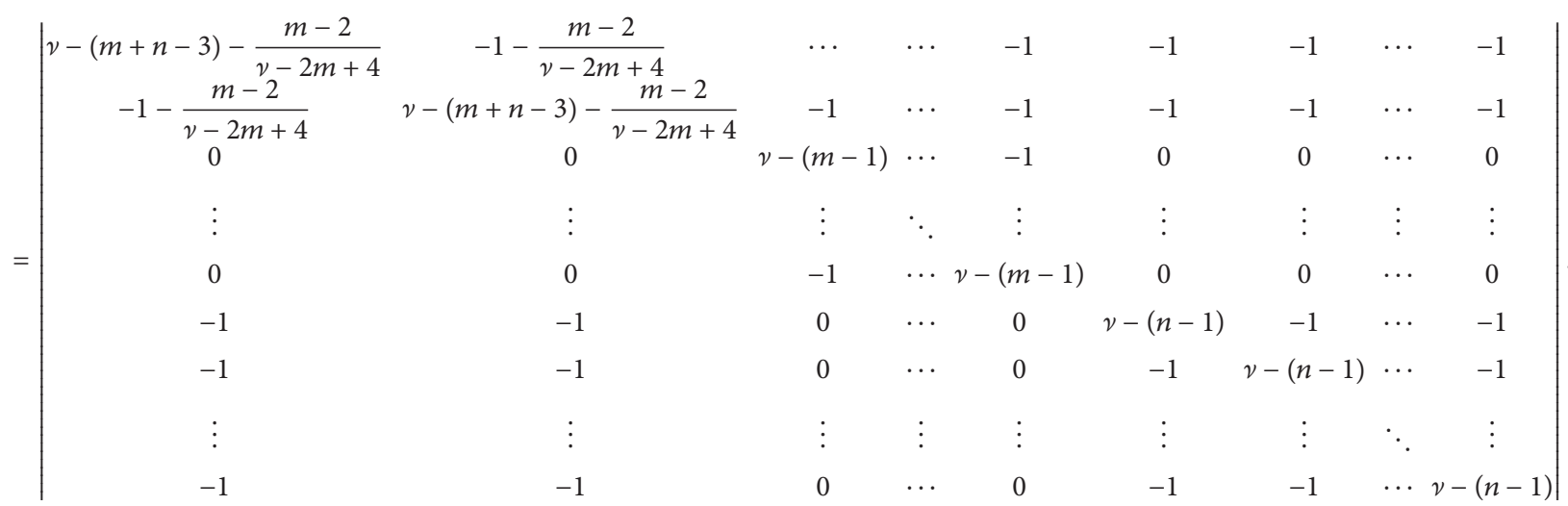

Again performing $C_{1}, C_{2}+(1 /(\nu-2)) \sum_{j=3}^{m+n-2} C_{j}$ and expanding directly yield

$$
\begin{aligned}
& \left|\nu I-Q\left(K_{m}{ }^{\circ} K_{n}\right)\right|=Q\left(K_{m-2}: v\right) Q\left(K_{n-2}: v\right) \\
& .\left|\begin{array}{cc}
\nu-(m+n-3)-\frac{m-2}{v-(2 m+4)}-\frac{n-2}{v-(2 n+4)} & -1-\frac{m-2}{v-(2 m+4)}-\frac{n-2}{v-(2 n+4)} \\
-1-\frac{m-2}{v-(2 m+4)}-\frac{n-2}{v-(2 n+4)} & v-(m+n-3)-\frac{m-2}{v-(2 m+4)}-\frac{n-2}{v-(2 n+4)}
\end{array}\right| .
\end{aligned}
$$

On performing elementary operations, we finally arrive at

$$
\begin{aligned}
\mid \nu I & -Q\left(K_{m}{ }^{\circ} K_{n}\right) \mid \\
& =[\nu-(m+n-4)][\nu-(m-2)]^{m-3}[\nu-(n-2)]^{n-3}\left[\nu^{3}\right. \\
& -(3 m+3 n-10) v^{2}
\end{aligned}
$$

$$
\begin{aligned}
& +\left(2 m^{2}+n^{2}+4 m n-11 m-11 n+20\right) v \\
& -4(m+n-4)(m-2)(n-2)] .
\end{aligned}
$$

On equating to zero and extracting eigenvalues from the equation above, the theorem follows. 
Corollary 13. From Lemma 7, the energy (adjacency) of line graph of $K_{m}{ }^{\circ} K_{n}$ is given by

$$
\begin{aligned}
E\left[L\left(K_{m}{ }^{\circ} K_{n}\right)\right]= & \left|m^{2}+n^{2}-3 m-3 n+2\right| \\
& +|m+n-6|+|m-4|(m-3) \\
& +|n-4|(n-3)+\left|\lambda_{1}\right|+\left|\lambda_{2}\right| \\
& +\left|\lambda_{3}\right|
\end{aligned}
$$

where, $\lambda_{1}, \lambda_{2}$, and $\lambda_{3}$ are the roots of the cubic equation:

$$
\begin{aligned}
\lambda^{3}- & (3 m+3 n-16) \lambda^{2} \\
& +\left(2 m^{2}+2 n^{2}+8 m n-34 m-34 n+92\right) \lambda \\
& +4\left(m^{2}+n^{2}+4 m n-14 m-14 n+32\right) \\
& -(m+n-4)(m n-2 m-2 n+4)=0 .
\end{aligned}
$$

Corollary 14. From Lemma 8, the energy (adjacency) of subdivision graph of $K_{m}{ }^{\circ} K_{n}$ where $m, n \geq 2$ is given by

$$
\begin{aligned}
E\left[S\left(K_{m} \circ e_{n}\right)\right]= & 2 \sqrt{m+n-4}+2 \sqrt{m-2}(m-3) \\
& +2 \sqrt{n-2}(n-3)+\left|\sqrt{\lambda_{1}}\right|+\left|\sqrt{\lambda_{2}}\right| \\
& +\left|\sqrt{\lambda_{3}}\right|,
\end{aligned}
$$

where $\lambda_{1}, \lambda_{2}$, and $\lambda_{3}$ are the roots of the equation

$$
\begin{aligned}
& \lambda^{3}-(3 m+3 n-10) \lambda^{2} \\
& \quad+2\left(m^{2}+n^{2}+4 m n-11 m-11 n+20\right) \lambda \\
& \quad-4(m+n-4)(m-2)(n-2)=0 .
\end{aligned}
$$

\section{Competing Interests}

The authors declare that they have no competing interests.

\section{References}

[1] D. M. Cvetković, M. Doob, and H. Sachs, Spectra of Graphs, vol. 87 of Pure and Applied Mathematics, Academic Press, New York, NY, USA, 1980.

[2] I. Gutman, "The energy of a graph," Berichte der MathematischStatistischen Sektion im Forschungszentrum Graz, vol. 103, pp. $1-22,1978$.

[3] R. Balkrishnan, “The energy of a graph," Linear Algebra and its Applications, vol. 387, pp. 287-295, 2004.

[4] D. Stevanović, "Energy and NEPS of graphs," Linear and Multilinear Algebra, vol. 53, no. 1, pp. 67-74, 2005.

[5] J. Anderson and T. D. Morley, "Eigenvalues of the Laplacian of a graph," Linear and Multilinear Algebra, vol. 18, no. 2, pp. 141-145, 1985.

[6] B. Mohar, "The Laplacian spectrum of graphs," in Graph Theory, Combinatorics, and Applications, vol. 2, pp. 871-898, 1988.
[7] B. Zhou, I. Gutman, and T. Aleksić, "A note on Laplacian energy of graphs," MATCH Communications in Mathematical and in Computer Chemistry, vol. 60, no. 2, pp. 441-446, 2008.

[8] R. Grone and R. Merris, "The Laplacian spectrum of a graph II," SIAM Journal on Discrete Mathematics, vol. 7, no. 2, pp. 221-229, 1994.

[9] R. Grone, R. Merris, and V. S. Sunder, "The Laplacian spectrum of a graph," SIAM Journal on Matrix Analysis and Applications, vol. 11, no. 2, pp. 218-238, 1990.

[10] R. Merris, "Laplacian matrices of graphs: a survey," Linear Algebra and Its Applications, vol. 197-198, pp. 143-176, 1994.

[11] R. Merris, “A survey of graph Laplacians," Linear and Multilinear Algebra, vol. 39, no. 1-2, pp. 19-31, 1995.

[12] N. Abreu, D. M. Cardoso, I. Gutman, E. A. Martins, and M. Robbiano, "Bounds for the signless laplacian energy," Linear Algebra and Its Applications, vol. 435, no. 10, pp. 2365-2374, 2011.

[13] D. Cvetković, "Signless Laplacians and line graphs," Bulletin Classe des Sciences Mathématiques et Naturelles. Sciences Mathématiques, vol. 131, no. 30, pp. 85-92, 2005.

[14] D. Cvetkovic and S. K. Simic, "Towards a spectral theory of graphs based on the Signless Laplacian I," Publications de l'Institut Mathématique, vol. 85, no. 99, pp. 19-33, 2009.

[15] D. Cvetković and S. K. Simić, "Towards a spectral theory of graphs based on the signless Laplacian, III," Applicable Analysis and Discrete Mathematics, vol. 4, no. 1, pp. 156-166, 2010.

[16] F. M. Dong, K. M. Koh, and K. L. Teo, Chromatic Polynomials and Chromaticity of Graphs, World Scientific Publishing, 2005.

[17] D. M. Cvetkovic, P. Rowlinson, and S. K. Simic, "Eigenvalue bounds for the signless Laplacian," Publications de l'Institut Mathématique, vol. 81, no. 95, pp. 11-27, 2007.

[18] D. M. Cvetkovic, M. Doob, and H. Sachs, Spectra of Graphs, Johann Ambrosius Barth, Heidelberg, Germany, 3rd edition, 1995. 


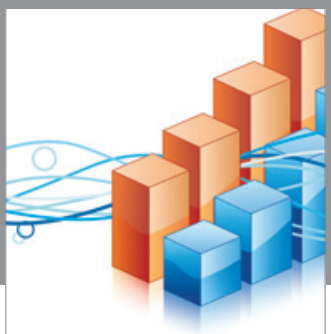

Advances in

Operations Research

vatem alat4

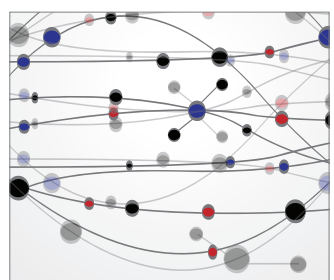

\section{The Scientific} World Journal
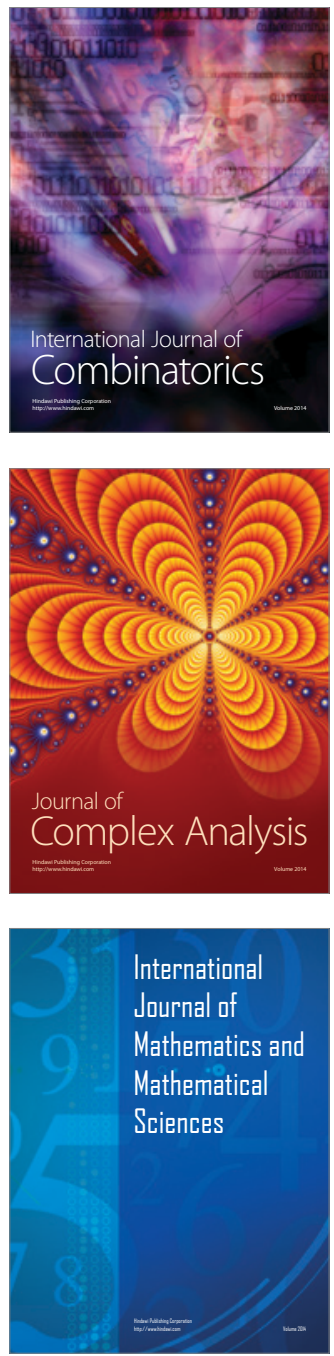
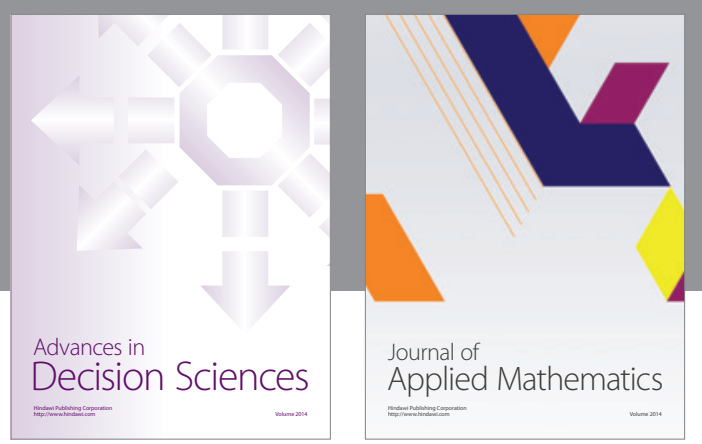

Algebra

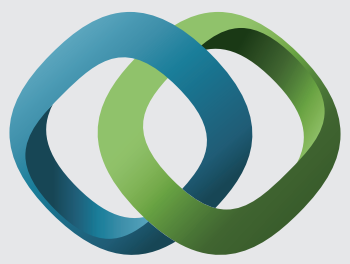

\section{Hindawi}

Submit your manuscripts at

http://www.hindawi.com
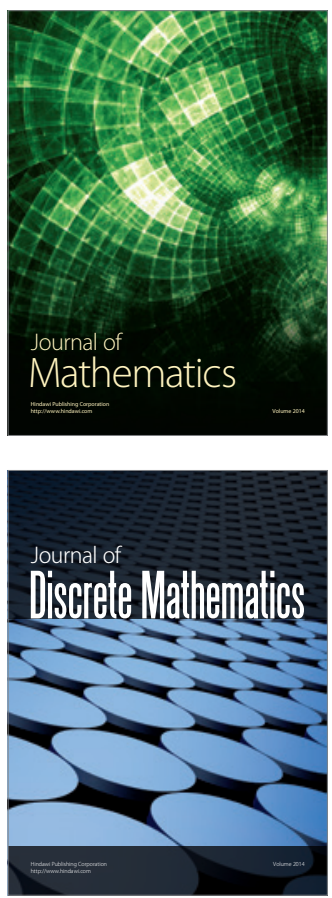

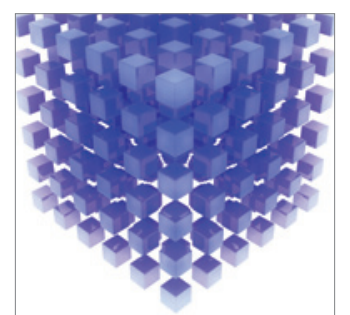

Mathematical Problems in Engineering
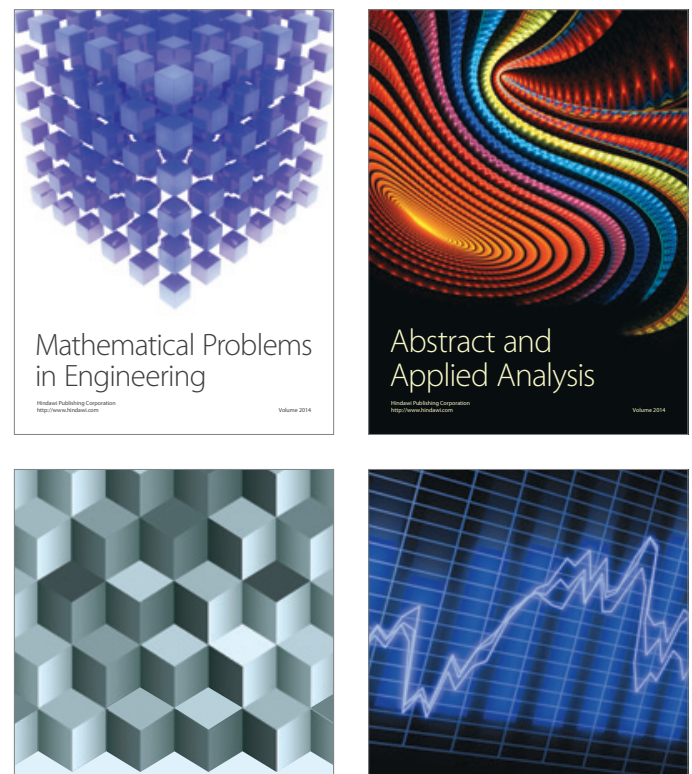

Journal of

Function Spaces

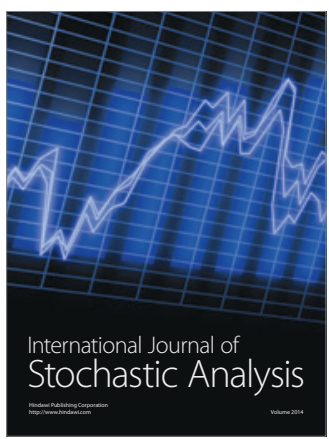

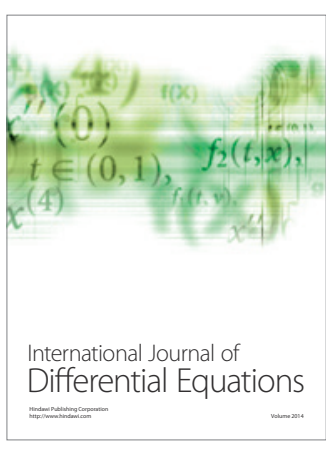
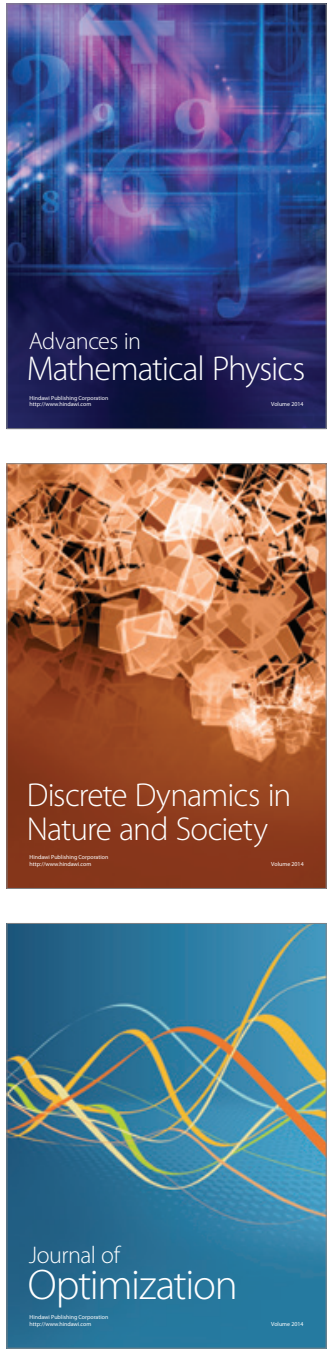